\title{
Long-term MRA follow-up after coiling of intracranial aneurysms: impact on mood and anxiety
}

\author{
Sandra P. Ferns $\cdot$ Pythia T. Nieuwkerk • \\ Willem Jan J. van Rooij • Gabriël J. E. Rinkel • \\ Charles B. L. M. Majoie
}

Received: 2 March 2010 /Accepted: 26 May 2010 / Published online: 23 June 2010

(C) The Author(s) 2010. This article is published with open access at Springerlink.com

\begin{abstract}
Introduction Magnetic resonance angiography (MRA) screening for recurrence of a coiled intracranial aneurysm and formation of new aneurysms long-term after coiling may induce anxiety and depression. In coiled patients, we evaluated effects on mood and level of anxiety from longterm follow-up MRA in comparison to general population norms.

Methods Of 162 patients participating in a long-term ( $>4.5$ years) MRA follow-up after coiling, 120 completed the EQ-5D questionnaire, a visual analog health scale and a self-developed screening related questionnaire at the time of MRA. Three months later, the same questionnaires were completed by 100 of these 120 patients. Results were compared to general population norms adjusted for gender and age.

Results Any problem with anxiety or depression was reported in 56 of 120 patients $(47 \%$; $95 \% \mathrm{CI} 38 \leftrightarrow 56 \%)$ at baseline and 42 of 100 patients $(42 \% ; 95 \% \mathrm{CI} 32 \leftrightarrow 52 \%)$ at 3 months, equally for screen-positives and -negatives. Compared to the reference population, participants scored $38 \%(95 \% \mathrm{CI} 967 \%)$ and $27 \%(95 \% \mathrm{CI} 4 \leftrightarrow 50 \%)$ more
\end{abstract}

S. P. Ferns $(\bowtie) \cdot$ P. T. Nieuwkerk · C. B. L. M. Majoie

Department of Radiology, Academic Medical Center,

Meibergdreef 9,

1105 AZ Amsterdam, The Netherlands

e-mail: S.P.Ferns@amc.uva.nl

W. J. J. van Rooij

Department of Radiology, St. Elisabeth Ziekenhuis,

Tilburg, The Netherlands

G. J. E. Rinkel

Department of Neurology, Rudolf Magnus Institute

of Neuroscience, University Medical Center,

Utrecht, The Netherlands often any problem with anxiety or depression. Three months after screening, $21 \%$ (20 of 92) of screennegatives and $13 \%$ (one of eight) of screen-positives reported to be less afraid of subarachnoid hemorrhage (SAH) compared to before screening. One of eight screenpositives reported increased fear of SAH.

Conclusions Patients with coiled intracranial aneurysms participating in long-term MRA screening reported significantly more often to be anxious or depressed than a reference group. Screening did not significantly increase anxiety or depression temporarily. However, subjectively, patients did report an increase in anxiety caused by screening, which decreased after 3 months.

Keywords Coiling · Magnetic resonance angiography (MRA) · Screening $\cdot$ EQ-5D questionnaire · Anxiety

\begin{tabular}{ll}
\multicolumn{2}{l}{ Abbreviations } \\
MRA & magnetic resonance angiography \\
SAH & subarachnoid hemorrhage \\
MRI & magnetic resonance imaging \\
TOF MRA & time-of-flight MRA \\
VAS & visual analog scale
\end{tabular}

Introduction

Extended imaging follow-up of coiled aneurysms that are adequately occluded at 6 months is under debate because the yield of clinically relevant findings appears to be low [1-3]. Although imaging follow-up with magnetic resonance angiography (MRA) has a negligible risk of complications, a follow-up regime longer than necessary may have a negative impact on the quality of life of these 
patients. Results from screening programs in patients with breast cancer and abdominal aortic aneurysms have indicated that potential benefits of a positive result of screening may be negated by reduced quality of life $[4,5]$. On the other hand, a screen-negative result may increase psychosocial wellbeing [5].

A previous study has shown that patients with a history of subarachnoid hemorrhage (SAH) and neurosurgical clipping have higher hospital anxiety and depression scale scores than a reference group [6]. Anxiety seems an important factor in assessing cost effectiveness of longterm follow-up screening after neurosurgical aneurysm clipping. In a cost-effectiveness study on long-term screening after clipping of aneurysms, screening did not increase quality adjusted life years in general, but would do so if patients had a modest decreased quality of life from fear for a recurrence [7].

The purpose of our study was to evaluate the impact on anxiety and depression of long-term (more than 4.5 years) MRA screening in patients with a coiled intracranial aneurysm that was adequately occluded at 6 months follow-up. We assessed anxiety and depression scores directly after screening, before the results of the scan were notified to the patient; and 3 months later, a possible shortterm increase in anxiety as a result of the screening would be neutralized by 3 months [8]. We compared both scores with scores of the general Dutch population. In addition, we aimed to compare anxiety and depression scores of patients with a history of SAH to patients without a history of SAH.

\section{Methods}

\section{Selection of patients}

In the Netherlands, an ongoing multi-center study called the LOng Term follow Up Study of coiled intracranial aneurysms (LOTUS) started in 2005. In this study, the occlusion status of coiled intracranial aneurysms is evaluated with long-term MRA after coiling. Review boards of the participating centers approved the study protocol. Inclusion criteria for invitation to participate to LOTUS were: coiling of an intracranial aneurysm more than 4.5 years earlier with adequate aneurysm occlusion on 6 months follow-up angiography, current age under 70 years, and no contra-indication for magnetic resonance imaging (MRI). MRA was performed on a 3 Tesla scanner without contrast administration [1].

Although the main purpose of the LOTUS study is assessment of the occlusion status of the coiled aneurysm, also the formation of new aneurysms and growth of additional untreated aneurysms are evaluated. Participants are informed about the low risk of finding either an incompletely occluded aneurysm or a newly formed or grown aneurysm, but if found, treatment or further followup must be considered in view of the risk of SAH. Within 4 weeks after the scan, participants are informed about the scan results by telephone or at the outpatient clinic.

From November 2007 to July 2009, all participants of the LOTUS study were invited to fill out a questionnaire directly after the MRA. The questionnaire had to be returned by mail within 4 weeks, before the MRA scan results were notified to the patient. Patients who responded in time received a second questionnaire by mail at least 3 months after the MRA. If the second questionnaire was not returned within 2 months, patients received another copy of the second questionnaire by mail.

\section{Questionnaires}

The questionnaire consisted of four parts. The first part was a list of questions about patient history of SAH and family history of SAH. Second, we used validated Dutch versions of the EuroQol (EQ-5D) that contain five questions addressing mobility, usual activities, pain, self-care, and anxiety and depression, and a visual analog scale where persons can rate their overall health state ranging from 0 (worst imaginable health state) to 100 (best imaginable health state). Third was a self-developed list of screening related questions (Fig. 1). In this list, patients were asked about the frequency of thoughts about the coiled aneurysm, about fear for a (recurrent) SAH and about sleep deprivation because of worrying in relation to the aneurysm. Answers were indicated on a Likert scale ranging from 'always' to 'never'. For each question, the patient was asked if participation in this study caused more arousal, choosing 'yes' or 'no'. Patients were asked how they felt about the participation to the screening ranging from 'very content' to 'regret'. Finally, patients were asked if they wanted to be screened again the next year and in 5 years.

\section{Data analysis}

The scores of the anxiety and depression question of the EQ-5D questionnaire directly after screening and at 3 months follow-up was compared to reference data from 851 subjects in a sample survey from Rotterdam, the Netherlands, matched for gender and age [9]. The proportion of participants reporting any problem in the anxiety and depression at baseline and at 3 months were compared to the reference group as absolute difference in proportion with $95 \%$ confidence interval (CI). The mean baseline EQVAS scores were compared to 3 months with a paired sample $t$ test. Answers to the screening related questions in the Likert scale were described and reduced to a nominal level 'agree' and 'disagree' to compare between baseline and follow-up measurements using $\chi^{2}$ tests. 
Fig. 1 Patients' response to the screening related questions at MRI screening and after 3 months

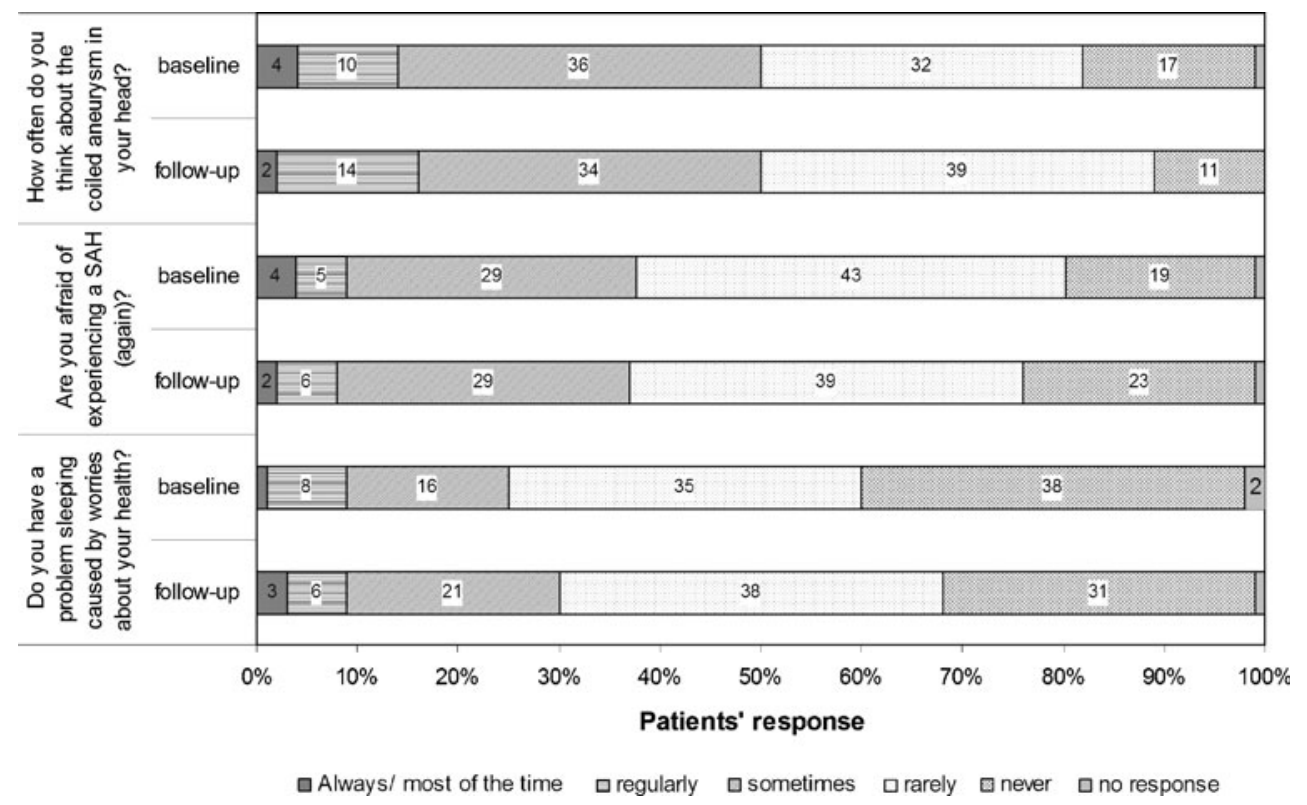

Anxiety and depression scores from patients with a history of a SAH were compared between baseline and at 3 months and with patients without SAH with Fisher's exact test. Responses to the screening-related questions were compared between the two groups using $\chi^{2}$ and Fisher's exact test. Data from screen-positive patients were separately described. For the EQ-5D data-analyses, all returned questionnaires at baseline and at 3 months were included. For all other comparisons of data from baseline with 3 months questionnaires, only the data of patients with two completed questionnaires was assessed.

\section{Results}

\section{Patient characteristics}

Between September 2007 and June 2009, 312 patients were invited to participate in the LOTUS study, and 162 patients were enrolled to undergo an MRA scan more than 4.5 years after coiling of an intracranial aneurysm. Reasons for patients not to participate in the LOTUS study are summarized in Fig. 2.

Of 162 invited patients, $120(74 \%)$ returned the completed questionnaire in time, and 42 patients did not want to fill out the questionnaire or were too late returning it. The second questionnaire was sent at least 3 months after the MRI scan to the 120 patients that completed the first one and was returned by $100(83 \%)$ patients mean 8.3 months (range 2.9-20.4 months) after the MRI scan.

Of 120 included patients, 82 were female $(68 \%)$. Mean age was 55 years (median 56, range 23-70 years). Of 120 patients, $106(88 \%)$ had a ruptured aneurysm that was coiled and 14 $(12 \%)$ were coiled for an unruptured aneurysm. Fourteen patients $(12 \%)$ had a family history of intracranial aneurysms.
EQ-5D

In the baseline questionnaire, 56 of 120 patients (47\%; 95\% CI $38-56 \%$ ) reported to have any problem with anxiety or depression; and at 3 months, this was reported by 42 of 100 patients $(42 \%, 95 \%$ CI $32-52 \%$; Fig. 3 Table 1$)$. If the analysis is restricted to the subset of 100 patients who completed the questionnaires at both assessments, the proportions of patients with any problem with anxiety or depression were $44 \%$ (95\% CI $34-54 \%)$ at time of MRA and $42 \%(95 \%$ CI $32-52 \%) 3$ months after MRA (difference $2 \%$; $95 \% \mathrm{CI}$ of difference -1 to $4 \%$ ) This indicates that anxiety or depression was not significantly increased directly after screening compared to 3-months

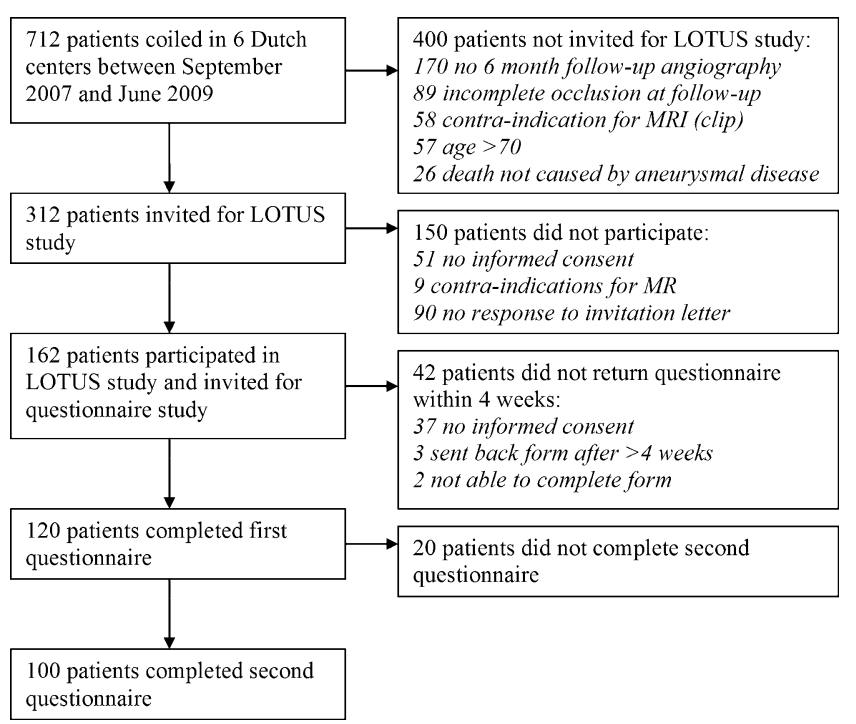

Fig. 2 Flowchart showing patient selection for the LOTUS study and the present sub-study 


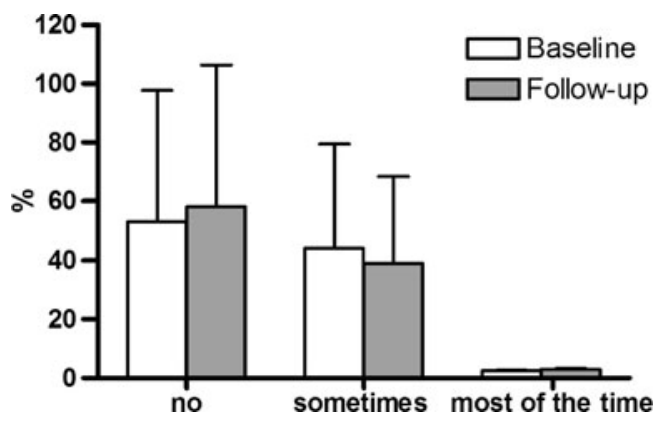

Fig. 3 Patients' response to "Are you anxious or depressed?" question at MRI screening and after 3 months

follow-up ( $p=0.89)$. However, compared to the $19 \%(95 \%$ CI $16-22 \%$ ) in the general Dutch population and adjusted for gender and age, patients reported 38\% (95\% CI: 9-67\%) more often to have 'any problem' with anxiety or depression in the baseline questionnaire and $27 \%$ (95\% CI $4-50 \%$ ) more often at 3 months (Fig. 4). Both proportions were significantly higher $(p<0.001)$.

\section{EQ-VAS}

Overall health in 100 patients that completed both questionnaires was rated mean 75 points out of 100 (median 79.5, range 4-100). At 3 months, this was with 72.1 points (median 75 , range 10-100), which was significantly lower $(p=0.03)$ (Table 1$)$.

\section{Screening-related questions}

Answers to screening-related questions at baseline and at 3 months are shown in Fig. 1.

At time of MRA, 14 of 99 patients (14\%) indicated to think regularly or most of the time of the coiled aneurysm; and at 3 months, this was 16 of 100 (16\%). To the question 'Did participation in this study increase frequency of these thoughts?', 26 of 93 (28\%) of patients confirmed at baseline and seven of $100(7 \%)$ at 3 months; three of these seven patients had a recurrent or new aneurysm.

Of 99 patients, $91(92 \%)$ responded not to be afraid of a (recurrent) SAH and 85 of 96 patients (91\%) felt that participation in the study did not increase this fear. At 3 months, 21 patients reported to be less afraid of an SAH after the screening. Of the two patients reporting increased fear for a SAH at 3 months, in one patient an aneurysm recurrence was found.

Of 98 patients, 89 (91\%) indicated not to sleeping disturbances because of worries of their state of health and participation on screening did not alter this in 91 of 100 patients (91\%). At 3 months, nine patients reported to sleep better and two to sleep worse after screening. In these two patients, an aneurysm recurrence was found.
At 3 months, 89 of 100 participants (89\%) were content or very content with participation on the screening; no patients regretted participation. Seventy-nine participants (79\%) were interested in a yearly screening and $89(89 \%)$ in screening every 5 years.

Patients with a history of SAH versus patients without SAH

Of 100 patients who completed both questionnaires, 88 had a history of SAH and 12 had not. Forty-one of $88 \mathrm{SAH}$ patients $(47 \%)$ had any problem with anxiety or depression at baseline; and at 3 months, this was reported by 37 patients $(42 \%),(p=$ $0.50)$. Three of $12(25 \%)$ non-SAH patients had any problem with anxiety of depression at the time of MRA, a nonsignificantly lower proportion compared to SAH patients $(p=0.16)$. At 3 months, five of $12(42 \%)$ non-SAH patients reported to have any problem with anxiety or depression; this increase was not significant $(p=0.67)$.

Of $82 \mathrm{SAH}$ patients that responded, $25(30 \%)$ reported an increase in frequency of thoughts about the coiled aneurysm due to screening compared to one of 11 responding non-SAH patients $(9 \%)$. This difference was significant $(p=0.04)$. However, at 3 months, this difference was no longer present with six of $88 \mathrm{SAH}$ patients $(7 \%)$ and one of 11 responding non-SAH patients (9\%) reporting an increase in thoughts due to screening. Screening caused more fear for a recurrent $\mathrm{SAH}$ at baseline in SAH patients (nine of 84 respondents, 11\%) compared to no non-SAH patients $(0 \%)$; but at 3 months, this difference was no longer present. To the question about disturbed sleep because of worries about health, SAH patients and nonSAH patients responded comparable.

Screen-positive patients

In eight of 100 patients who completed both questionnaires, a new finding was present on MRI. All eight patients had a history of SAH and two had familial intracranial

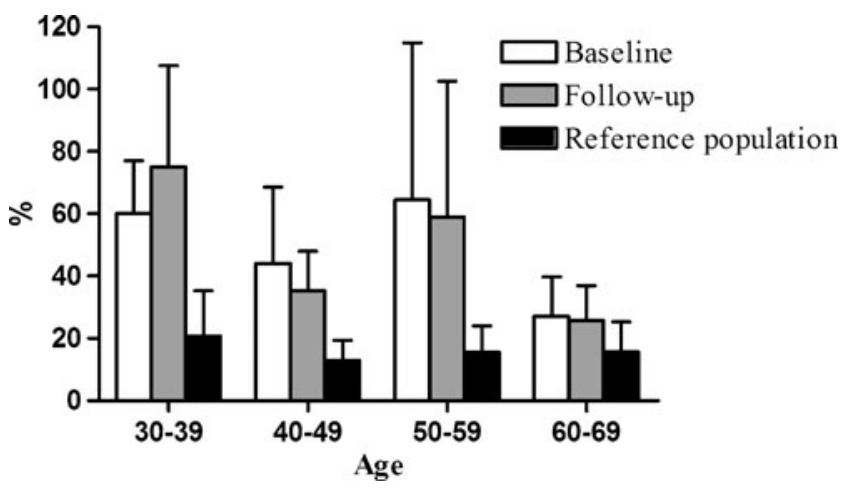

Fig. 4 Patients reporting to have 'any problem' with anxiety or depression in the EQ-5D questionnaire at MRI screening and after 3 months compared to Dutch general population norms 
aneurysms. Four patients needed additional treatment for a recurrence or de novo aneurysm, three patients had small aneurysm recurrences for which extended follow-up was recommended, and one patient had a small recurrence that needed no further follow-up. At 3 months, three of eight $(38 \%)$ screen-positive patients reported 'any problem' with anxiety, compared to 39 of 92 (42\%) screen negatives. Two screen-positive patients scored more anxiety or depression at 3 months than at baseline. All eight screen positives were very content with the participation in the study.

\section{Discussion}

In this study, we found that patients with a coiled intracranial aneurysm that were screened with MRI more than 4.5 years after coiling are more anxious or depressed at the time of screening and 3 months thereafter compared to Dutch general population norms. Half of the patients reported to have a problem with anxiety or depression at the time of screening. Screening did not significantly increase anxiety or depression temporarily. However, a quarter of the patients indicated that screening caused more frequent thinking about the coiled aneurysm and one in ten reported to be more afraid for recurrent $\mathrm{SAH}$, even before results of screening were communicated. Three months after screening, one in seven patients reported that screening had decreased thoughts of their coiled aneurysm and one in five was less afraid of a recurrent $\mathrm{SAH}$.

The EQ-5D questionnaire we used is a rough tool and cannot assess a moderate temporary increase in anxiety or depression caused by screening in our population. However, our study does rule out a major impact of long-term follow-up screening on quality of life and in particular on anxiety or depression. Our screening-related questions are not validated, but may imply subjective feelings of temporary increased fear in participants. A negative screening result, although positive news for the patient, did not substantially decrease anxiety or depression, but provided a subjective feeling of relieve in most participants and no patients regretted participation. Surprisingly, a screen-positive result did not influence quality of life, nor did it cause a subjective feeling of increased fear for SAH in most patients. Compared to patients without a history of $\mathrm{SAH}$, patients with a history of SAH are more anxious at the time of long-term follow-up screening.

In a comparable long-term follow-up screening study of patients with a ruptured aneurysm that was surgically clipped, patients were also more anxious or depressed than a general population group [6].

Our study has several limitations. First, we only invited participants of our multi-center long-term follow-up study after coiling (LOTUS) to fill out the questionnaires. Participation in LOTUS indicated an a priori interest in screening. We did not invite the patients who declined screening, either out of lack of interest or out of fear for the screening or its results. If fear of screening would be a major reason to decline screening, our study is likely to underestimate the true anxiety scores in patients long-term after coiling. However, the goal of this study was to assess impact on mood and anxiety of patients that participate in a long-term follow-up screening; hence, non-participants are not exposed to this additional burden. Second, although the same questionnaires were filled out at the time of MRI and 3 months later in order to pick up a possible temporary increase in anxiety induced by the screening, our study lacks a true baseline measurement and we have no solid proof that temporarily increased anxiety will have resided within 3 months. If this duration is longer, we have measured too short [8]. Third, although this is the largest study to assess anxiety in this specific patient group to date, our sample size was too small to compare different subgroups, for example patients with and without familial intracranial aneurysms and screen-positives and screennegatives. In our comparison of patients with or without a history of SAH, we encountered large confidence intervals because of the small sample of patients without a history of SAH.

Table 1 Patient characteristics, EQ-5D anxiety/depression scores and VAS scores of participants compared to Dutch general population norms.

\begin{tabular}{lllll}
\hline & $\begin{array}{l}\text { Baseline } \\
(n=120)\end{array}$ & $\begin{array}{l}\text { Follow-up } \\
(n=100)\end{array}$ & $\begin{array}{l}\text { Reference group } \\
(n=851)\end{array}$ & $\begin{array}{l}\text { Screen-positives at follow-up } \\
(n=8)\end{array}$ \\
\hline Number of men & $38(32 \%)$ & $33(33 \%)$ & $384(45 \%)$ & $1(13 \%)$ \\
Mean age (median, range) & $55(56,23-70)$ & $55(57,23-70)$ & $-{ }^{\mathrm{d}}$ & $56(57,46-63)$ \\
Number with anxiety or depression $^{\mathrm{b}}$ & $56(47 \%)$ & $42(42 \%)$ & $161(19 \%)$ & $3(38 \%)$ \\
Mean VAS $^{\mathrm{c}}$ & 75.0 & 72.1 & 80.3 & 59.0 \\
\hline
\end{tabular}

\footnotetext{
${ }^{\text {a }}$ Reference group from Essink-Bot et al. [9] published in Health Economics in 1993

b Number of participants reporting to have 'any problem' with anxiety or depression in the Dutch EQ-5D questionnaire

${ }^{\mathrm{c}}$ VAS from the EQ-5D questionnaire with scores from 0 to 100 , with a score of 0 being the worst and 100 the best imaginable health state

${ }^{\mathrm{d}}$ Patient age is reported per decade; $13 \%=18-29$ years, $19 \%=30-39$ years, $13 \%=40-49$ years, $12 \%=50-59$ years, $16 \%=60-69$ years, $17 \%=70-79$ years, $10 \%=80+$ years
} 
Anxiety is a very important factor in assessing costeffectiveness of long-term follow-up screening after neurosurgical aneurysm clipping [7]. To our knowledge, our study is the first to assess psychosocial impact of longterm MRA screening in patients with a history of intracranial aneurysm coiling. Screening did not temporarily increase anxiety, nor did a negative screening result give a substantial decrease in anxiety. Aneurysms that are coiled with adequate occlusion at 6 months follow-up are likely to remain sufficiently occluded at long-term followup [1]. In addition, the frequency of new aneurysm formation and enlargement of untreated aneurysms in this patient population appears to be low [3]. Clinical relevance of long-term screening of patients with adequately occluded coiled aneurysms is doubtful [10], emphasized by our study results that patients with a coiled intracranial aneurysm are more anxious compared to a reference group.

\section{Conclusion}

Patients with a history of aneurysm coiling and especially patients with a history of SAH and coiling are more anxious or depressed compared to a Dutch general population group. Long-term follow-up screening does not induce a major increase in anxiety or depression temporarily, and a negative screening outcome does not give a substantial decrease in the patients' feelings of anxiety or depression. The decision to subject patients with a coiled intracranial aneurysm to long-term follow-up should be made considering clinical relevance only, and follow-up is not recommended for the sole purpose of reassuring the patient.

Acknowledgment This work was supported by a grant from the Nuts Ohra Foundation, the Netherlands.

Conflict of interest statement We declare that we have no conflict of interest.

Open Access This article is distributed under the terms of the Creative Commons Attribution Noncommercial License which per- mits any noncommercial use, distribution, and reproduction in any medium, provided the original author(s) and source are credited.

\section{References}

1. Sprengers ME, Schaafsma J, van Rooij WJ, Sluzewski M, Rinkel GJ, Velthuis BK, van Rijn JC, Majoie CB (2008) Stability of intracranial aneurysms adequately occluded 6 months after coiling: a 3T MR angiography multicenter long-term follow-up study. AJNR Am J Neuroradiol 29:17681774

2. Schaafsma JD, Sprengers ME, van Rooij WJ, Sluzewski M, Majoie CB, Wermer MJ, Rinkel GJ (2009) Long-term recurrent subarachnoid hemorrhage after adequate coiling versus clipping of ruptured intracranial aneurysms. Stroke 40:1758-1763

3. Sprengers ME, van Rooij WJ, Sluzewski M, Rinkel GJ, Velthuis BK, de Kort GA, Majoie CB (2009) MR angiography follow-up 5 years after coiling: frequency of new aneurysms and enlargement of untreated aneurysms. AJNR Am J Neuroradiol 30:303307

4. Lindholt JS, Vammen S, Fasting H, Henneberg EW (2000) Psychological consequences of screening for addominal aortic aneurysm and conservative treatment of small abdominal aortic aneurysms. Eur J Vasc Endovasc Surg 20:7983

5. Scaf-Klomp W, Sanderman R, van de Wiel HB, Otter R, van den Heuvel WJ (1997) Distressed or relieved? Psychological side effects of breast cancer screening in the Netherlands. J Epidemiol Community Health 51:705-710

6. Wermer MJ, Kool H, Albrecht KW, Rinkel GJ (2007) Aneurysm Screening after Treatment for Ruptured Aneurysms Study Group. Subarachnoid hemorrhage treated with clipping: long-term effects on employment, relationships, personality and mood. Neurosurgery 60:91-98

7. Wermer MJ, Koffijberg H, van der Schaaf IC, ASTRA study Group (2008) Effectiveness and cost of screening for aneurysms every 5 years after subarachnoid hemorrhage. Neurology 27:2053-2062

8. Simon AE, Steptoe A, Wardle J (2005) Socioeconomic status differences in coping with a stressful medical procedure. Psychosom Med 67:270-276

9. Essink-Bot ML, Stouthard MEA, Bonsel GJ (1993) Generalizability of valuations on health states collected with the EuroQol ${ }^{\mathrm{c}}$ questionnaire. Health Econ 2:237-246

10. van Rooij WJ, Sluzewski M (2009) Opinion: imaging follow-up after coiling of intracranial aneurysms. AJNR Am J Neuroradiol 30:1646-1648 